\title{
Relationship between Structural Remodeling and Reactivity in Pulmonary Resistance Arteries from Hypertensive Piglets
}

\author{
DAVID A. KELLY, ALISON A. HISLOP, SUSAN M. HALL, AND SHEILA G. HAWORTH \\ Vascular Biology and Pharmacology Unit, Institute of Child Health, University College London, \\ London WC1N 1EH, UK
}

\begin{abstract}
In neonatal pulmonary hypertension, the pulmonary arteries fail to adapt to extrauterine life and remain thick walled. In a previous study on normal neonatal resistance arteries, perfusion myography and confocal microscopy showed that responses to agonist stimulation were related to wall structure. We hypothesized that in hypertensive resistance pulmonary arteries, an enhanced response to contractile and relaxant agonist stimulation would be associated with an increased wall thickness and abnormal postnatal cytoskeletal remodeling of smooth muscle cells (SMC). Pulmonary arteries (110-140 $\mu \mathrm{m}$ external diameter) from normal piglets and those exposed to chronic hypobaric hypoxia from birth or from $3 \mathrm{~d}$ of age were mounted on a perfusion myograph. Lumen diameter and SMC nuclear positions were tracked after addition of $\mathrm{KCl}$, the thromboxane mimetic U46619, and bradykinin. After fixation in situ, SMC dimensions were measured using confocal and electron microscopy. In all hypertensive animals, wall thickness and SMC density were increased and SMC length/width ratio decreased.
\end{abstract}

\section{ABSTRACT}

After hypoxic exposure for $3 \mathrm{~d}$, arteries from animals exposed from birth showed a greater and faster contractile response than controls, but arteries from piglets first exposed at $3 \mathrm{~d}$ of age did not, though both showed similar structural appearance. Increase of exposure to $11 \mathrm{~d}$ elicited an enhanced response and further cytoskeletal remodeling. All vessels relaxed fully to bradykinin. SMC remodeling and reactivity appear to be influenced by the age at onset and the duration of the hypoxic insult. (Pediatr Res 58: 525-530, 2005)

Abbreviations
F-actin, fibrillar actin
PGF $_{2 \alpha}$, prostaglandin F2 $\alpha$
PPHN, persistent pulmonary hypertension of the newborn
SMC, smooth muscle cell
U46619, thromboxane mimetic
$\boldsymbol{\alpha}$-SM actin, $\alpha$-smooth muscle actin

Rapid structural and functional changes occur in the pulmonary arteries as pulmonary vascular resistance decreases after birth (1). Thick-walled fetal arteries remodel to thin-walled structures within days of birth (2). Using perfusion myography in conjunction with confocal microscopy we found that postnatal elongation of SMC within resistance arteries was associated with a decrease in the speed and extent of vessel constriction in response to agonist stimulation (3).

Structural studies on the pulmonary vessels of babies with PPHN and in piglets with experimental PPHN (4-6) show an increase in pulmonary artery medial thickness and the maturation of SMC in normally nonmuscular precapillary vessels (6). Whereas babies with PPHN appear to have an excessively reactive pulmonary arterial circulation, pharmacological stud-

Received July 13, 2004; accepted January 26, 2005.

Correspondence: A. Hislop, Ph.D., Vascular Biology and Pharmacology Unit, Institute of Child Health, 30 Guilford St., London WC1N 1EH, UK; e-mail: a.hislop@ich.ucl.ac.uk This work was funded by the British Heart Foundation.

DOI: 10.1203/01.pdr.0000176928.54471.07 ies on conduit arteries from pulmonary hypertensive piglets showed that there was no increase in the contractile response to potassium chloride $(\mathrm{KCl}), \mathrm{PGF} 2 \alpha$, or U46619, although the relaxation response to acetylcholine was impaired $(7,8)$. We believed that the resistance pulmonary arteries would show an enhanced response to contractile stimulation, and that the response would be related to the structural abnormalities.

In the present study, we hypothesized that the SMC in the thickened wall of resistance arteries from pulmonary hypertensive piglets would show an increase in myofilaments and abnormal cytoskeletal organization in association with an enhanced response to agonist stimulation. We studied pulmonary resistance arteries from neonatal piglets that had been exposed to hypobaric hypoxia, using a perfusion myograph to assess reactivity and confocal and transmission electron microscopy to describe SMC cell density, shape, size and cytoskeletal organization in the same arteries. We studied piglets exposed from 20 min of age for $3 \mathrm{~d}$ and compared these animals with those exposed for $3 \mathrm{~d}$ after a $3 \mathrm{~d}$ period of normal adaptation in room air. To study the effect of a longer period of exposure 
to hypoxia, we studied animals exposed for $11 \mathrm{~d}$ from $3 \mathrm{~d}$ of age. Findings were compared with those in age matched controls.

\section{MATERIALS AND METHODS}

Origin of tissue. Tissue was obtained from normal Large White piglets, which were killed at birth ( $<5 \mathrm{~min})$ and 3,6 , and $14 \mathrm{~d}$ of age $(n \geq 9$ per age group). To induce pulmonary hypertension, piglets were exposed to chronic hypobaric hypoxia $(50.8 \mathrm{KPa})(8)$ either from birth $(<20 \mathrm{~min})$ to $3 \mathrm{~d}$ of age $(3 \mathrm{~d}$ hypoxia), or starting at $3 \mathrm{~d}$ of age for 3 (6d hypoxia) or $11 \mathrm{~d}$ (14d hypoxia), $n$ $\geq 8$ in each age group. Previous studies have shown that piglets treated in this manner develop pulmonary hypertension, evidenced by right ventricular hypertrophy and an increase in pulmonary arterial smooth muscle $(6,8)$. All hypoxic animals were killed immediately on removal from the hypobaric chamber. All animals received humane care in compliance with the British Home Office Regulations and the Principles of Laboratory Animal Care formulated by the National Society of Medical Research and the Guide for the Care and Use of Laboratory Animals published by the National Institutes of Health [Publication No. (National Institutes of Health) 85-23, Revised 1996, Office of Science and Health Reports, DRR/National Institutes of Health, Betheseda, MD 20892].

Functional studies. Perfusion myograph studies were carried out as previously described (3). Briefly peripheral pulmonary arteries immediately proximal to the alveolar region (approx. $1.5 \mathrm{~mm}$ in length) were dissected free of the surrounding parenchyma and mounted on a perfusion myograph (LSI, Burlington, VT) (9). Vessels were orientated to the in vivo direction of flow and bathed in PSS at $37^{\circ} \mathrm{C}$ bubbled with $95 \% \mathrm{O}_{2}, 5 \% \mathrm{CO}_{2}$. Nuclei were fluorescently labeled with Hoechst 33258 in situ on the myograph. To monitor changes in lumen diameter vessels were imaged continuously using a CCD camera (Alrad Instruments Ltd., Newbury, UK) and measurements were recorded on a Powerlab (ADInstruments Ltd., Hastings, UK). Changes in position of fluorescently labeled SMC nuclei were recorded using a separate camera after addition of each agonist (3). Images were stored using Openlab 3 (Improvision, Coventry, UK).

Vessels (two per animal) were equilibrated for $45 \mathrm{~min}$ in PSS at a constant flow of $30 \mu \mathrm{L} / \mathrm{min}$ and then brought to a maintained pressure of either 20 or $45 \mathrm{~mm} \mathrm{Hg}$ to approximate to a normal or hypertensive pulmonary artery pressure. Extraluminal $\mathrm{KCl}(125 \mathrm{mM})$ was applied and the vessel allowed to contract fully as determined by no further change in lumen diameter. After a washout with PSS, when the vessel returns to baseline, extraluminal U46619 $(2 \mu \mathrm{M})$ was added. The concentration of U46619 was based on previously published dose response studies on pulmonary arteries from normal and hypertensive piglets $(3,7)$. A bolus of $50 \mu \mathrm{L}$ of intraluminal bradykinin $(1 \mu \mathrm{M})$ was then applied, and the vessel allowed to fully relax. In a previous dose response study, this dose of bradykinin induced maximal relaxation in porcine resistance arteries from fetal to adult life, the $\mathrm{ED}_{50}$ being similar in all preparations (10). The time taken to achieve maximal contraction or relaxation for each agonist was noted.

To relate change in lumen diameter to the response of individual SMC, the position of the fluorescently labeled SMC nuclei was tracked by recording X-Y coordinates for the centre of each nucleus before and after each agonist stimulation, as previously described (3).

Structural studies. At the end of the functional study, vessels were fixed while still pressurized in the myograph, either at the point of maximal relaxation to bradykinin or at maximal contraction (3). Nonstimulated vessels from each age group were also fixed at 20 or $45 \mathrm{~mm} \mathrm{Hg}$ pressure for study of baseline appearance. Half of each vessel was embedded for cryosectioning and confocal microscopy, and half was postfixed in $1 \%$ glutaraldehyde and prepared for transmission electron microscopy.

Confocal microscopy. Twenty-micrometer-thick frozen sections of arteries were cut in transverse section, perpendicular to the vessels' longitudinal axis. After permeabilization and blocking for nonspecific proteins, they were incubated with anti- $\alpha$-SM actin (Sigma Chemical Co., Poole, UK) or phalloidin conjugated to Alexa 568 (Sigma Chemical Co.) to label F-actin. Staining was visualized with rabbit anti-mouse IgG TRITC conjugate. Cell nuclei were labelled using YOYO-1 or TO-PRO-3 (Molecular Probes, Eugene, OR) for the $\alpha$-SM actin- and phalloidin-labeled sections, respectively. Washed sections were mounted in Vectamount (Vector Laboratories, Peterborough, UK) for confocal microscopy (MRC-600 or Radiance 2000, Bio-Rad, UK). Z-stacks of 20 confocal images were acquired over a thickness of $20 \mu \mathrm{m}$ and from these a three-dimensional image of each artery was created using Confocal Assistant 4.02. From these images, a longitudinal section of each SMC showing the maximal cross-sectional area was obtained from which the area, length, and width of the cell was measured using Openlab 3 image analysis software. A mean area and a mean length/width ratio were calculated for each group of normal and pulmonary hypertensive animals, in both the relaxed and contracted state.

Transmission electron microscopy. Transverse $1-\mu \mathrm{m}$ sections were cut perpendicular to the vessels' longitudinal axis and labeled with toluidine blue ( $n>5$ for each group of animals). In each section, the medial wall thickness was measured, the number of SMC nuclei in the media counted, and the cell density per unit area of media calculated. Ultrathin sections of all these vessels were examined using a Jeol $100 \mathrm{CX}$ electron microscope. From each artery a low-power image (magnification $\times 33,000$ ) of the full thickness of the wall was taken at two points on opposite sides of the vessel. A total of at least 20 images per group of animals were measured by planimetry and areas of SMC, elastin, collagen, and the remaining intercellular space within the media was quantified (Openlab 3). From these, the proportion of each component was calculated.

Statistical analysis. Change in lumen diameter, internuclear distance, response time, wall thickness, SMC area, density and length/width ratio, and volume proportions of wall components were analyzed using one-way ANOVA followed by a post hoc Tukey's test (Minitab). $p<0.05$ was considered statistically significant. Comparisons were first made between results obtained for each group of animals at maintained perfusion pressures of 20 and $45 \mathrm{~mm} \mathrm{Hg}$. No difference was observed in any of the studies so the values at the two pressures were pooled. Comparisons were made between data from the hypertensive animals and their age matched controls at the age at which the hypertensive animals were put into and taken out of the hypobaric chamber.

\section{RESULTS}

Confirmation of pulmonary hypertension. After death, the hearts were dissected and weighed and the ratio of the weight of the left ventricle plus septum to the right ventricle was calculated. For all three groups exposed to hypobaric hypoxia there was a significant decrease in ratio indicating right ventricular hypertrophy in all hypoxic animals compared with age matched controls ( $3 \mathrm{~d}$ hypoxia, $1.53 \pm 0.06$ versus $1.91 \pm 0.1$; $6 \mathrm{~d}$ hypoxia, $1.73 \pm 0.07$ versus $2.37 \pm 0.07 ; 14 \mathrm{~d}$ hypoxia, 1.72 \pm 0.14 versus $3.01 \pm 0.14 ; p<0.05$ for each comparison). Thus, all hypoxic animals were pulmonary hypertensive.

Functional studies performed on the perfusion myograph. After dissection, arteries from all groups of animals had an unpressurized external diameter of between 110 and $140 \mu \mathrm{m}$. Each vessel had two to three SMC layers in the media.

Changes in lumen diameter in response to agonist stimulation. In normal arteries, $\mathrm{U} 46619$ and $\mathrm{KCl}$ caused a reduction in lumen diameter, the vessels were vasoconstricted. The response was greatest at birth, decreased by $3 \mathrm{~d}$ of age and remained low at 6 and $14 \mathrm{~d}$ (Fig. 1 a). Both $\mathrm{KCl}$ and $\mathrm{U} 46619$ induced vasoconstriction was greater in arteries from 3d hypoxia animals compared with the normal at $3 \mathrm{~d}(p<0.05$ for both, Fig. 1a) In animals exposed from $3 \mathrm{~d}$ of age, $6 \mathrm{~d}$ hypoxia arteries did not show an increase in vasoconstriction compared with age-matched controls but $14 \mathrm{~d}$ hypoxia arteries did ( $p<$ 0.05 for U46619, Fig. 1a). Pulmonary arteries from $3 \mathrm{~d}$ hypoxia and $14 \mathrm{~d}$ hypoxia animals contracted more rapidly than those from age matched controls ( $46 \pm 5$ versus $106 \pm 13 \mathrm{~s}$ for $\mathrm{U} 46619$ at $3 \mathrm{~d}$ and $58 \pm 7$ versus $156 \pm 15 \mathrm{~s}$ at $14 \mathrm{~d} ; p<0.05$ for both comparisons).

On addition of bradykinin to fully contracted normal and hypertensive arteries, all relaxed back almost to the baseline lumen diameter (Fig. 1a). In arteries from normal animals, the time taken to relax was similar at all ages and similar to the time taken to contract to U46619. The relaxation was faster than normal in $3 \mathrm{~d}$ hypoxia $(52 \pm 4$ versus $102 \pm 13 \mathrm{~s} ; p<$ 0.05 ) but normal in $6 \mathrm{~d}$ hypoxia and $14 \mathrm{~d}$ hypoxia arteries. 
a

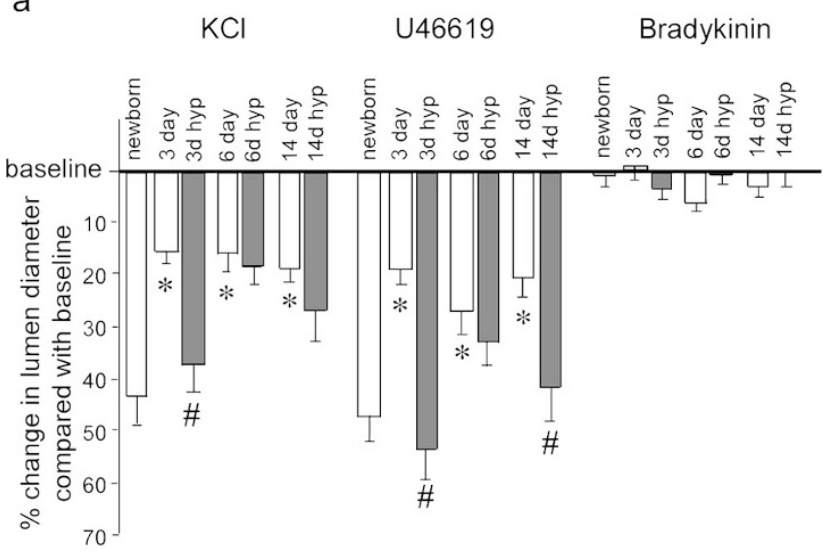

b

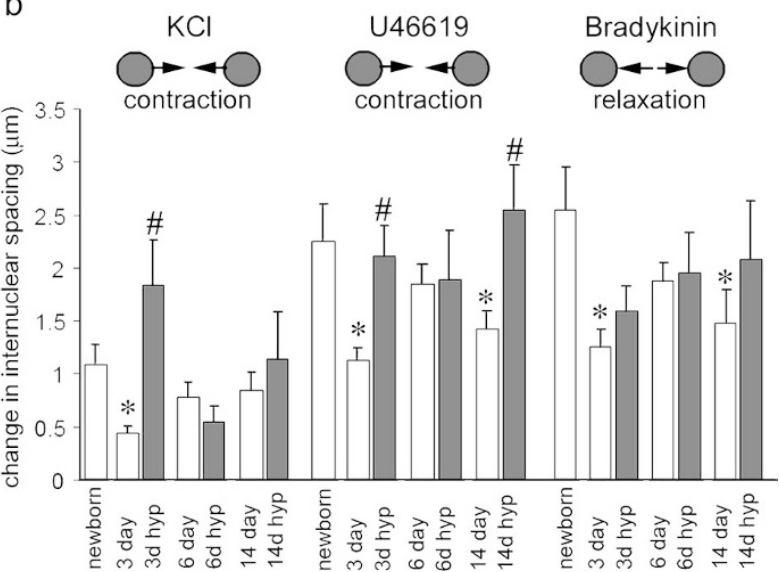

Figure 1. (a) Percentage decrease in lumen diameter (mean \pm SEM), $n \geq 12$ arteries per group) from baseline tone ( $x$ axis) in response to KCl and $\mathrm{U} 46619$ and the return to close to baseline diameter on relaxation after addition of bradykinin. (b) Change in smooth muscle cell internuclear distance (mean \pm SEM), $n \geq 12$ arteries per group) on addition of $\mathrm{KCl}, \mathrm{U} 46619$, and bradykinin, in resistance arteries from normal and hypoxic pulmonary hypertensive piglets. One-way ANOVA with post hoc Tukey's tests using Minitab Release 12. ${ }^{*} p<0.05$ compared with newborn, $\# p<0.05$ compared with normal age-matched controls.

Change in spatial relationship of SMC nuclei. In all arteries, the distance between two adjacent nuclei decreased on contraction to $\mathrm{KCl}$ and $\mathrm{U} 46619$ and increased on relaxation to bradykinin. In normal arteries, U46619 induced a significantly greater reduction in internuclear distance in the newborn arteries than in arteries from $3 \mathrm{~d}$ and $14 \mathrm{~d}$ old animals $(p<0.05$, Fig. 1 b). For $\mathrm{KCl}$, the pattern of response was similar but less marked. In arteries from $3 \mathrm{~d}$ hypoxia animals, the reduction in internuclear distance was greater than in age-matched controls in response to both $\mathrm{KCl}$ and $\mathrm{U} 46619$ ( $p<0.05$ for both comparisons, Fig. 1b). In animals exposed from $3 \mathrm{~d}$ of age, $6 \mathrm{~d}$ hypoxia arteries showed a similar reduction in nuclear spacing to age-matched controls. This reduction was greater than normal in 14d hypoxia arteries ( $p<0.05$ for U46619, Fig. $1 b$ ). On exposure to bradykinin, the nuclei of all contracted arteries moved apart to regain their basal position (Fig. 1b).

In both normal and hypertensive arteries, changes in internuclear distance reflected the changes in total lumen diameter.

Structure of the vessel wall. Within each normal and hypertensive group of animals there was no significant difference in arterial wall thickness or SMC density measured on tolu- idine blue-stained sections from contracted, relaxed, and unstimulated arteries. Thus, the values were pooled for each group. In normal animals, the wall thickness and SMC density decreased with age. The mean wall thickness was greater in the pulmonary arteries from all hypertensive animals than in agematched controls ( $p<0.05$ for each comparison, Table 1$)$, and was similar to the normal newborn value. The density of SMC within the wall was greater in all the hypertensive vessels than in age-matched controls $(p<0.05$ for each comparison, Table $1)$ and in $14 \mathrm{~d}$ hypoxia animals was higher than in the normal newborn $(p<0.05)$.

Measurements of smooth muscle cell dimensions from confocal images were similar in $\alpha$-SM actin- and phalloidinstained sections. For both the contracted and relaxed state, the maximal cross-sectional area of normal, individual SMC increased after birth, reaching significance at $14 \mathrm{~d}$ in the former and at $6 \mathrm{~d}$ in the latter (Table 1). The maximal cross-sectional area of SMC from hypertensive arteries was significantly less than that from age matched controls at all ages (Table 1). Both contracted and relaxed cells in all hypertensive arteries had a significantly smaller length/width ratio suggesting a less elon-

Table 1. Influence of hypoxic pulmonary hypertension on the structure of pulmonary resistance arteries and smooth muscle cells (mean $\pm S E M)$

\begin{tabular}{|c|c|c|c|c|c|c|c|}
\hline Age & Newborn & \multicolumn{2}{|c|}{$3 \mathrm{~d}$} & \multicolumn{2}{|c|}{$6 \mathrm{~d}$} & \multicolumn{2}{|c|}{$14 \mathrm{~d}$} \\
\hline WT (m) & $21.3 \pm 1.4$ & $11.5 \pm 1.0^{*}$ & $19.2 \pm 2.2 \#$ & $9.6 \pm 0.7 *$ & $20.0 \pm 1.1 \#$ & $10.8 \pm 1.1^{*}$ & $18.4 \pm 1.3 \#$ \\
\hline SMC density $\left(\right.$ cells $\left./ \mu \mathrm{m}^{2} \times 1000\right)$ & $12.7 \pm 0.9$ & $7.8 \pm 0.5^{*}$ & $15.2 \pm 2.0 \#$ & $9.4 \pm 0.8^{*}$ & $13.8 \pm 1.6 \#$ & $4.4 \pm 0.2^{*}$ & $22.6 \pm 1.7 \# *$ \\
\hline No. & 7 & 7 & 6 & 5 & 6 & 5 & 9 \\
\hline Area of each SMC contracted $\left(\mu \mathrm{m}^{2}\right)$ & $34.5 \pm 1.7$ & $41.5 \pm 4.2$ & $25.9 \pm 1.1 \#$ & $45.2 \pm 4.7$ & $30.2 \pm 1.4 \#$ & $55.2 \pm 2.9^{*}$ & $24.7 \pm 4.2 \#$ \\
\hline No. & 7 & 11 & 9 & 6 & 6 & 9 & 7 \\
\hline Length/width contracted (U46619) & $4.5 \pm 0.2$ & $4.0 \pm 0.2$ & $2.8 \pm 0.2 \#$ & $3.4 \pm 0.3 *$ & $2.8 \pm 0.2 \#$ & $4.2 \pm 0.3$ & $2.2 \pm 0.1 \#$ \\
\hline No. & 7 & 8 & 6 & 9 & 6 & 10 & 3 \\
\hline Length/width relaxed (bradykinin) & $4.9 \pm 0.2$ & $4.9 \pm 0.2$ & $4.0 \pm 0.2 \# \dagger$ & $4.8 \pm 0.3 \dagger$ & $4.0 \pm 0.3 \# \dagger$ & $6.8 \pm 0.4^{* \dagger}$ & $2.7 \pm 0.2 \#$ \\
\hline No. & 8 & 10 & 7 & 8 & 9 & 8 & 9 \\
\hline
\end{tabular}

WT and SMC density data pooled from unstimulated, contracted, and relaxed vessels there being no significant difference between groups.

$* p<0.05$ compared with newborn animals; \# $p<0.05$ compared with age matched normal animals; $\dagger p<0.05$ between contracted and relaxed values. 
gated, more rounded shape than in age matched controls (Table 1). The length/width ratio of smooth muscle cells in arteries of both normal and hypertensive animals was smaller after contraction to U46619 than after relaxation to bradykinin (Table $1)$.

Smooth muscle cell structure. By confocal microscopy, bundles of actin fibers labeled with phalloidin (F-actin) and $\alpha$-SM actin were visible in the cytoplasm of all medial smooth muscle cells in the arteries from all normal animals. Smooth muscle cells from normal 3-, 6-, and 14-d-old animals had long actin bundles oriented parallel to the cells' longitudinal axis, but in newborn and in hypoxic animals, these appeared shorter and had variable orientation (Fig. 2 a). In three-dimensional reconstructions, it could be seen that the actin fibers formed a meshwork throughout each cell. This appearance was confirmed by transmission electron microscopy where myofilament bundles appeared thicker and less well orientated to the longitudinal axis and occupied a greater proportion of the SMC cytoplasm than normal (Fig. 3). $\alpha$-SM actin labeled fiber bundles in all SMC from all animals except the 14d hypoxia

a

\section{3 day normal}
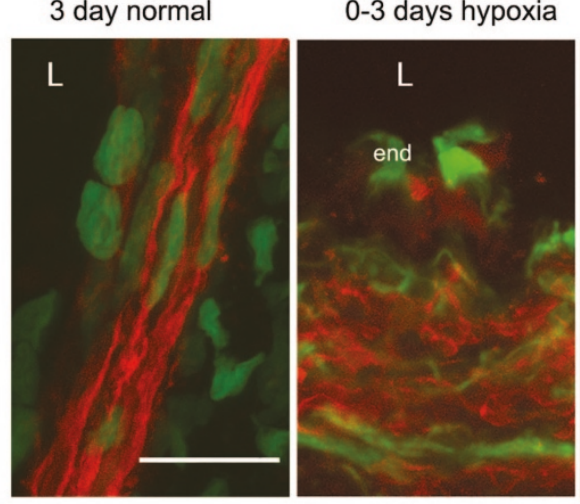

\section{3-6 days hypoxia}

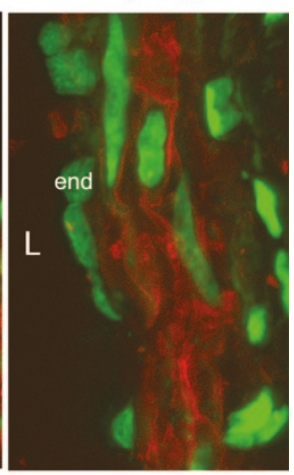

b

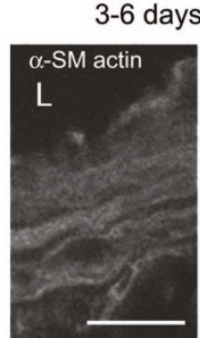

3-14 days hypoxia
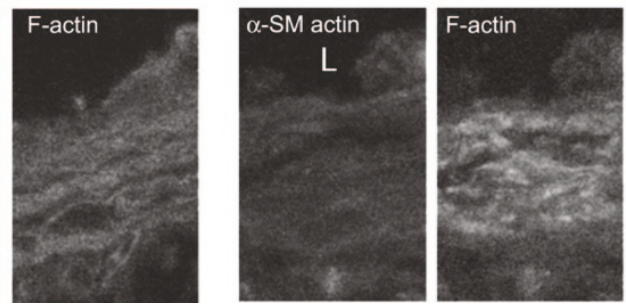

Figure 2. Confocal images of $20 \mu \mathrm{m}$ transverse cryosections of resistance arteries. (a) In sections stained for $\alpha$-smooth muscle actin (red) and with YOYO-1 (green), normal 3-d-old piglets have elongated oval nuclei and bundles of actin fibers lie parallel to the cells longitudinal axis. In the artery from a $3 \mathrm{~d}$ hypoxia piglet ( $0-3 \mathrm{~d}$ hypoxia), the nuclei appear distorted and actin bundles are short with varying orientation throughout the cytoplasm and form a meshwork of fibers within each cell. In $6 \mathrm{~d}$ hypoxia (3-6 d hypoxia), cells the fusiform nuclei are surrounded by a meshwork of actin fiber bundles. end, endothelium. Bar represents $25 \mu \mathrm{m}$ for all images. (b) Sections of arteries from $6 \mathrm{~d}$ hypoxia (3-6 d hypoxia) and 14d hypoxia (3-14 d hypoxia) piglets double labeled for $\alpha$-SM actin and F-actin (phalloidin). In 6d hypoxia cells, there is extensive co-localization of $\alpha$-SM actin (excitation wavelength of $488 \mathrm{~nm}$ ) with F-actin (excitation wavelength of $568 \mathrm{~nm}$ ). In 14d hypoxia cells, there was no clear co-localization of $\alpha$-SM actin and the thick F-actin bundles and $\alpha$-SM actin is seen as weak diffuse stain throughout the cytoplasm. Bar represents $25 \mu \mathrm{m}$ for all images.

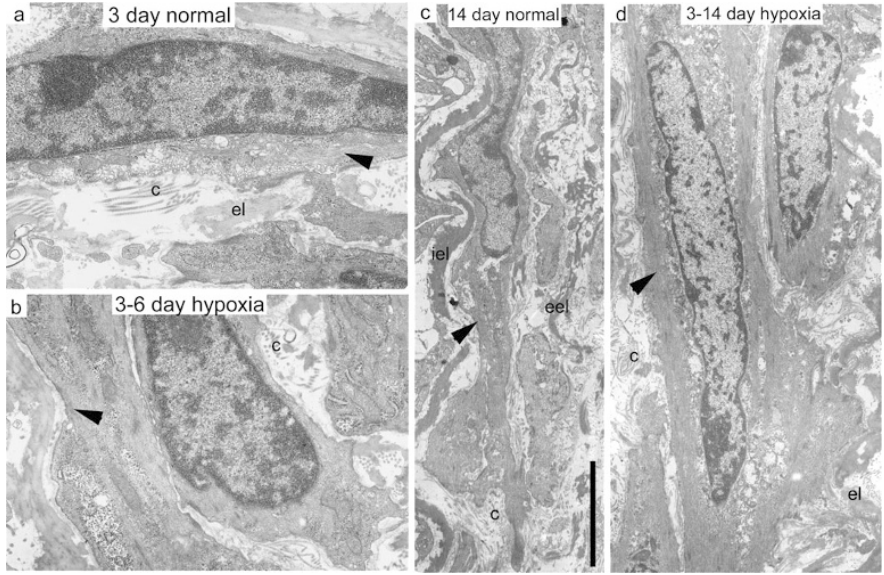

Figure 3. Transmission electron micrographs of pulmonary resistance arteries from normal and hypertensive piglets. In the artery from normal 3-d $(a)$ and 14-d-old $(c)$ piglets, SMC are circumferentially oriented and sheets of myofilaments (arrowheads) lie parallel to the cells longitudinal axis. SMC are loosely packed and the extracellular matrix components elastin $(e l)$ and collagen fibres $(c)$ are surrounded by unoccupied space. In the arteries from hypertensive piglets, $6 \mathrm{~d}$ hypoxia $(b)$ and $14 \mathrm{~d}$ hypoxia $(d)$ adjacent SMC have differing orientations and contain thick sheets and bundles of myofilaments. SMC are tightly packed and there is less unoccupied space than in vessels from normal piglets. eel, external elastic lamina; iel, internal elastic lamina. Bar represents $5 \mu \mathrm{m}$ for all images.

animals. In these, $\alpha$-SM actin labeling was only detectable as a weak diffuse stain throughout the cell cytoplasm (Fig. 2b)

Area measurements of arterial wall composition made on transmission electron micrographs (SMC, elastin, collagen, and intercellular space) revealed a significant increase in the percentage area of media occupied by SMC in the 3d hypoxia and $14 \mathrm{~d}$ hypoxia animals compared with age-matched controls $(64.6 \pm 2.5$ and $57.9 \pm 3.4$ versus $44.5 \pm 2.6$ and $41.5 \pm 3.4$, respectively, $p<0.05$ for both comparisons). This change was associated with a decrease in percentage area occupied by intercellular space (18.6 \pm 1.9 and $21.1 \pm 2.3$ versus $28.2 \pm$ 1.8 and $36.5 \pm 2.1$, respectively, $p<0.05$ for both). The percentage area of muscle in the $6 \mathrm{~d}$ hypoxia animals was the same as in the normal animals. The proportion of collagen and elastin within the wall was similar in the normal and hypertensive arteries.

\section{DISCUSSION}

This is the first study of pulmonary hypertensive resistance arteries in which the response of individual SMC within the artery wall has been tracked after stimulation with contractile and relaxant agonists and then related to the changes in lumen diameter and wall structure. In normal immature arteries, we previously found that age-related changes in response to agonists could be attributed to the maturation of the vessel wall. Namely, a decrease in medial thickness at $14 \mathrm{~d}$ of age due to elongation of SMC with redistribution of actin fibres within SMC was associated with a smaller contractile response than occurred at birth (3). In the pulmonary hypertensive arteries, we expected that an increase in medial thickness and SMC hypertrophy with an increase in contractile myofilaments would be associated with enhanced contractile responsiveness. 
However, we did not find a consistent association between responsiveness and structure in the hypertensive arteries.

The techniques used in the present study were identical to those used previously to study normal arteries (3), and we used an intraluminal flow rate of $30 \mu \mathrm{L} / \mathrm{min}$ and two pressures, 20 and $45 \mathrm{~mm} \mathrm{Hg}$. The measurement $20 \mathrm{~mm} \mathrm{Hg}$ is close to the normal seen after birth when the pressure falls from 25 to 16 $\mathrm{mm} \mathrm{Hg}$ by $14 \mathrm{~d}$ (1) and $45 \mathrm{~mm} \mathrm{Hg}$ is a similar pressure to that recorded in hypoxic conditions in young piglets (11). However, the pressures used had little effect on the size or responsiveness of the arteries, whether normal or hypertensive. This contrasts with studies on mesenteric arteries $(12,13)$ where increased pressure was accompanied by an increase in lumen diameter. Pulmonary arteries may normally be responsive at lower pressures than systemic arteries and have already reached maximum tone at $20 \mathrm{~mm} \mathrm{Hg}$. Previous studies on adult pulmonary arteries showed that intrinsic contraction was present at $20 \mathrm{~mm}$ $\mathrm{Hg}$ (14).

A novel aspect of our experimental approach was to relate the changes in lumen diameter to changes in the spatial relationship of the SMC within the wall on agonist stimulation, and to relate these changes to SMC structure. Exposure to chronic hypobaric hypoxia for $3 \mathrm{~d}$ starting at birth enhanced the contractile response to both a receptor-dependent (U46619) and a receptor-independent $(\mathrm{KCl})$ agonist. The reduction in lumen diameter was greater than in normal arteries and this change was associated with a greater reduction in internuclear distance. The thickness of the vessel wall and the functional response remained similar to the normal at birth, suggesting failure to adapt to extrauterine life in a hypoxic environment (Fig. 4). However, the SMC within the wall were smaller, more rounded, and more closely packed than at birth. This suggests an active response to hypoxic conditions, not simply a failure to adapt. By contrast, when animals were exposed for $3 \mathrm{~d}$ after an initial $3 \mathrm{~d}$ period of adaptation in normoxia, the contractile response of the hypertensive pulmonary arteries was normal for age despite the arteries being abnormally thick walled and

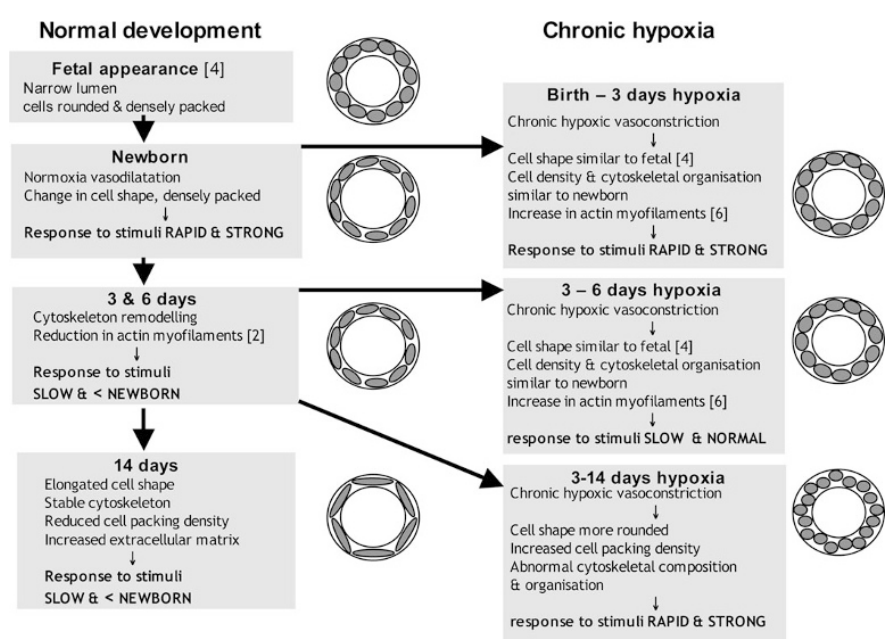

Figure 4. Diagram summarizing the findings of the present study and previously published observations on pulmonary resistance arteries from neonatal piglets. The structure and functional changes with age and development of hypertension are illustrated. Response to stimuli refers to contractile ( $\mathrm{KCl}$ and U46619) and relaxant (bradykinin) stimuli. resembling those of animals exposed from birth (Fig. 4). Other investigators studying muscular pulmonary arteries from piglets exposed to $11 \%$ oxygen from 2 to $3 \mathrm{~d}$ of age for $3-4 \mathrm{~d}$ also found no increase in responsiveness to U46619 (15). In the present study, however, when animals were exposed to hypoxia for a longer period of time, contraction was greater and more rapid. The response resembled that seen in animals exposed from birth but the SMC density was greater and the SMC had a distinctive phenotype. They showed abnormal cytoskeletal organization with loss of filamentous $\alpha$-SM actin and a further reduced length/width ratio and thus were different from the phenotype seen after 3 d hypoxia (Fig. 4). Greater changes in contractile reactivity followed a longer period of hypoxic exposure. However, the relaxant response to bradykinin was maintained. Other investigators found that a $10-\mathrm{d}$ period of hypoxic in piglets led to impaired nitric oxide function despite preserved levels of endothelial nitric oxide synthase (16).

During hypoxic exposure, the magnitude of the contractile response did not appear to be directly related to the intracellular organization of the actin cytoskeleton. After $3 \mathrm{~d}$ of hypoxia, whether from birth or $3 \mathrm{~d}$ of age, the density of myofilaments was abnormally increased as previously reported5 but contractility only increased following exposure from birth (Fig. 4). After exposure for $11 \mathrm{~d}$, a rapid and strong response to acute contractile stimuli developed but the increase in response was associated with loss of filamentous $\alpha$-SM actin indicating that cytoskeletal remodeling had continued in the hypoxic environment. This could have been due to sustained constriction over an $11 \mathrm{~d}$ period. In cultured SMC (A7r5) subjected to sustained contractile stimulus, disassembly of $\alpha$-actin filaments was found while $\beta$-actin was still present in dense cables (17). Phenotypic changes in response to hypoxia have been described previously in porcine, bovine, and rat conduit pulmonary arteries (18-20). In SMC isolated from conduit arteries of neonatal piglets exposed to $11 \mathrm{~d}$ hypoxia, a stable change in SMC phenotype, manifested by a change in cell shape and regulation of the actin cytoskeleton, accompanied altered functional responses (21). Conduit vessels are composed of heterogenous SMC populations, but we found little structural variation between cells in the walls of resistance arteries, confirming previous studies (2) showing that the pulmonary resistance arteries of calves also have a homogeneous SMC population (22). In the present study, the arterial walls of all hypertensive animals showed an increase in SMC density. In the younger animals this was probably due primarily to hypertrophy inasmuch as we did not find an increase in cell replication in earlier studies (23). However, replication may have contributed to the marked increase in SMC density seen in the $14 d$ hypoxic animals. In calves, an increase in multiplication was found after 4 and $7 \mathrm{~d}$ exposure to hypoxia from $2 \mathrm{~d}$ of age (19). Other potentially important alterations in SMC phenotype include hypoxia-induced changes in receptor density and function, in intracellular coupling mechanisms, and in the number of tension generating actin and myosin interactions, all of which could profoundly influence the response to agonist stimulation. 
We have focused on the relationship between reactivity and the structure of the arterial wall. But factors other than abnormalities in wall structure may have contributed to the altered reactivity. Prominent among these is the possibility of hypoxiainduced changes in receptor activation. For this study, we used a near-maximal dose of U46619 for both the control and hypertensive arteries based on previous studies on larger vessels to try to ensure full receptor occupancy. Nevertheless, the heightened response to the agonist at $14 \mathrm{~d}$ could perhaps be explained by a hypoxia-induced increase in receptor density rather than by a change in vessel wall structure. We cannot be sure that we are measuring maximal reactivity since a doseresponse curve to U46619 was not made on these hypertensive porcine pulmonary arteries. In the rat, an increase in contractile response after hypoxic exposure occurred without any change in the $\mathrm{EC}_{50}$ or increase in receptor number (24).

Our findings are consistent with the accepted view that the peripheral pulmonary arteries are the site of resistance, as in the systemic circulation (25) and they are extremely sensitive to hypoxia. We had previously shown that porcine pulmonary hypertensive conduit arteries do not show an enhanced response to either $\mathrm{KCl}$ or PGF2 $\alpha$ (8). Others have found that hypertensive adult rats have an increase in wall thickness but decreased reactivity to a variety of contractile agonists (26).

The present study confirms the clinical impression of excessive reactivity in the newborn. We had hypothesized that changes in wall structure and cell phenotype in response to hypoxia-induced hypertension would lead to a change in responsiveness. However, we found no consistent, simple relationship between pulmonary arterial structure and reactivity. An increase in wall thickness was not invariably associated with increased responsiveness. For the same period of hypoxic exposure and in the presence of similar structural change, reactivity was greater after exposure from birth than after an adaptive period in normoxia. A longer period of hypoxia was needed to elicit the increased functional response with greater SMC cytoskeletal remodeling. The novel approach to studying the relationship between reactivity and cell structure will make it possible to study relationships between receptor activation and cell signaling in experimental models of the developing vasculature in health and disease.

\section{REFERENCES}

1. Haworth SG, Hislop AA 1981 Adaptation of the pulmonary circulation to extrauterine life in the pig and its relevance to the human infant. Cardiovasc Res 15:108-119
2. Haworth SG, Hall SM, Chew M, Allen K 1987 Thinning of fetal pulmonary arterial wall and postnatal remodelling: ultrastructural studies on the respiratory unit arteries of the pig. Virchows Arch A Pathol Anat Histopathol 411:161-171

3. Kelly DA, Hislop AA, Hall SM, Haworth SG 2002 Correlation of pulmonary arterial smooth muscle structure and reactivity during adaptation to extrauterine life. J Vasc Res 39:30-40

4. Spitzer AR, Davis J, Clarke WT, Bernbaum J, Fox WW 1988 Pulmonary hypertension and persistent fetal circulation in the newborn. Clin Perinatol 15:389-413

5. Allen KM, Haworth SG 1986 Impaired adaptation of pulmonary circulation to extrauterine life in newborn pigs exposed to hypoxia: an ultrastructural study. J Pathol 150:205-212

6. Haworth SG, Hislop AA 1982 Effect of hypoxia on adaptation of the pulmonary circulation to extra-uterine life in the pig. Cardiovasc Res 16:293-303

7. Arrigoni FI, Hislop AA, Haworth SG, Mitchell JA 1999 Newborn intrapulmonary veins are more reactive than arteries in normal and hypertensive piglets. Am J Physiol 277:L887-L892

8. Tulloh RM, Hislop AA, Boels PJ, Deutsch J, Haworth SG 1997 Chronic hypoxia inhibits postnatal maturation of porcine intrapulmonary artery relaxation. Am J Physiol 272:H2436-H2445

9. VanBavel E, Mooij T, Giezeman MJ, Spaan JA 1990 Cannulation and continuous cross-sectional area measurement of small blood vessels. J Pharmacol Methods 24:219-227

10. Boels PJ, Deutsch J, Gao B, Haworth SG 1999 Maturation of the response to bradykinin in resistance and conduit pulmonary arteries. Cardiovasc Res 44:416-428

11. Scarborough JE, Daggett CW, Lodge AJ, Chai PJ, Williamson JA, Jaggers J, George SE, Ungerleider RM 1998 The role of endothelial nitric oxide synthase expression in the development of pulmonary hypertension in chronically hypoxic infant swine. J Thorac Cardiovasc Surg 115:343-348

12. Buus NH, VanBavel E, Mulvany MJ 1994 Differences in sensitivity of rat mesenteric small arteries to agonists when studied as ring preparations or as cannulated preparations. Br J Pharmacol 112:579-587

13. VanBavel E, Mulvany MJ 1994 Role of wall tension in the vasoconstrictor response of cannulated rat mesenteric small arteries. J Physiol 477:103-115

14. Liu Q, Wiener CM, Flavahan NA 1998 Superoxide and endothelium-dependent constriction to flow in porcine small pulmonary arteries. Br J Pharmacol 124:331-336

15. Fike CD, Kaplowitz MR, Pfister SL 2003 Arachidonic acid metabolites and an early stage of pulmonary hypertension in chronically hypoxic newborn pigs. Am J Physiol Lung Cell Mol Physiol 284:L316-L323

16. Fike CD, Aschner JL, Zhang Y, Kaplowitz MR 2004 Impaired NO signaling in small pulmonary arteries of chronically hypoxic newborn piglets. Am J Physiol Lung Cell Mol Physiol 286:L1244-L1254

17. Li C, Fultz ME, Parkash J, Rhoten WB, Wright GL $2001 \mathrm{Ca} 2+$-dependent actin remodeling in the contracting A7r5 cell. J Muscle Res Cell Motil 22:521-534

18. Meyrick B, Reid L 1979 Hypoxia and incorporation of $3 \mathrm{H}$-thymidine by cells of the rat pulmonary arteries and alveolar wall. Am J Pathol 96:51-70

19. Belknap JK, Orton EC, Ensley B, Tucker A, Stenmark KR 1997 Hypoxia increases bromodeoxyuridine labeling indices in bovine neonatal pulmonary arteries. Am J Respir Cell Mol Biol 16:366-371

20. Wohrley JD, Frid MG, Moiseeva EP, Orton EC, Belknap JK, Stenmark KR 1995 Hypoxia selectively induces proliferation in a specific subpopulation of smooth muscle cells in the bovine neonatal pulmonary arterial media. J Clin Invest 96:273281

21. Bailly K, Ridley AJ, Hall SM, Haworth SG 2004 RhoA activation by hypoxia in pulmonary arterial smooth muscle cells is age and site specific. Circ Res 94:13831391

22. Stiebellehner L, Frid MG, Reeves JT, Low RB, Gnanasekharan M, Stenmark KR 2003 Bovine distal pulmonary arterial media is composed of a uniform population of well-differentiated smooth muscle cells with low proliferative capabilities. Am J Physiol Lung Cell Mol Physiol 285:L819-L828

23. Hall SM, Hislop AA, Wu Z, Haworth SG 2004 Remodelling of the pulmonary arteries during recovery from pulmonary hypertension induced by neonatal hypoxia. J Pathol 203:575-583

24. Thomas BJ, Wanstall JC 2003 Alterations in pulmonary vascular function in rats exposed to intermittent hypoxia. Eur J Pharmacol 477:153-161

25. Pohl U, de Wit C, Gloe T 2000 Large arterioles in the control of blood flow: role of endothelium-dependent dilation. Acta Physiol Scand 168:505-510

26. Packer CS, Roepke JE, Oberlies NH, Rhoades RA 1998 Myosin isoform shifts and decreased reactivity in hypoxia-induced hypertensive pulmonary arterial muscle. Am J Physiol 274:L775-L785 